\title{
Morphological Instabilities in a Growing Yeast Colony: Experiment and Theory
}

Sams, Thomas; Sneppen, Kim; Jensen, Mogens; Ellegaard, Clive; Christensen, Bjørn Eggert; Thrane, Ulf

Published in:

Physical Review Letters

Link to article, DOI:

10.1103/PhysRevLett.79.313

Publication date:

1997

Document Version

Publisher's PDF, also known as Version of record

Link back to DTU Orbit

Citation (APA):

Sams, T., Sneppen, K., Jensen, M., Ellegaard, C., Christensen, B. E., \& Thrane, U. (1997). Morphological Instabilities in a Growing Yeast Colony: Experiment and Theory. Physical Review Letters, 79(2), 313-316. https://doi.org/10.1103/PhysRevLett.79.313

\section{General rights}

Copyright and moral rights for the publications made accessible in the public portal are retained by the authors and/or other copyright owners and it is a condition of accessing publications that users recognise and abide by the legal requirements associated with these rights.

- Users may download and print one copy of any publication from the public portal for the purpose of private study or research.

- You may not further distribute the material or use it for any profit-making activity or commercial gain

- You may freely distribute the URL identifying the publication in the public portal 


\title{
Morphological Instabilities in a Growing Yeast Colony: Experiment and Theory
}

\author{
Thomas Sams, Kim Sneppen, Mogens H. Jensen,* and Clive Ellegaard \\ Niels Bohr Institute and NORDITA, Blegdamsvej 17, DK-2100 Ø, Denmark \\ Bjørn Eggert Christensen \\ Novo Nordisk, Novo Allé 1, DK-2880 Bagsvard, Denmark \\ Ulf Thrane \\ Department of Biotechnology, Technical University of Denmark, DK-2800 Lyngby, Denmark
}

(Received 25 November 1996)

\begin{abstract}
We study the growth of colonies of the yeast Pichia membranaefaciens on agarose film. The growth conditions are controlled in a setup where nutrients are supplied through an agarose film suspended over a solution of nutrients. As the thickness of the agarose film is varied, the morphology of the front of the colony changes. The growth of the front is modeled by coupling it to a diffusive field of inhibitory metabolites. Qualitative agreement with experiments suggests that such a coupling is responsible for the observed instability of the front. [S0031-9007(97)03559-X]
\end{abstract}

PACS numbers: 87.10.+e, 87.15.-v, 87.22.As

The physical laws governing the development of biological morphologies have been under investigation even before Turing described pattern formation using interacting diffusive fields [1,2]. Turing patterns have found application in a variety of biological systems $[3,4]$. Lately the interplay between form and formation in biological morphologies has been investigated by physical modeling in swarms of swimming bacteria [5,6], in growth of bacteria colonies [7-12], and in growth of various fungi [13-15].

From a morphological point of view, the growth of fungi can be naturally divided into filamentous growth and yeastlike growth. Filamentous growth is characterized by a hyphal, multicellular, growth zone that under unfavorable growth conditions exhibits fractallike properties $[16,17]$. In contrast, yeasts are characterized by unicellular growth and compact colonies [18]. In the present Letter, the morphological properties of the front of yeast colonies are studied.

In the experiment, the yeast is grown on YPD with full strength defined as $10 \mathrm{~g} / 1$ yeast extract (Difco), $20 \mathrm{~g} / 1$ Bacto Peptone (Difco), $20 \mathrm{~g} / \mathrm{l}$ dextrose, $p \mathrm{H}$ unadjusted. Agarose (Sigma type II) is used for solidified media. The experiment is carried out by inoculating cells of the yeast Pichia membranaefaciens [19] along a thin stripe on the agarose film and monitoring the growth with a video camera. The temperature is held at $T=30{ }^{\circ} \mathrm{C}$ in a chamber with $100 \%$ relative humidity.

The typical size of a single yeast cell is $3 \times 5 \mu \mathrm{m}$. The velocity of the front is in the range $0.3-3 \mathrm{~mm} / \mathrm{h}$, depending on the thickness of the agarose film. With a typical doubling time of $1.6 \mathrm{~h}$, this means that a band of 200-2000 cells contributes to the growth of the front. In the spirit of Trinci, we call this band the optimal growth zone [20].

The yeast is immersed in a liquid which it produces and carries along as it grows. The surface tension of this carrier medium introduces a lower cutoff in the possible length scales for structures of the front.

In one experiment the yeast is grown on solid 0.5 strength YPD solidified with $0.8 \%$ agarose. Under these conditions the growth slows down during the experiment and virtually stops after about 4 days. Examples of fronts identified from digitized images of the growing culture are shown in Fig. 1(a) at $5 \mathrm{~h}$ intervals.

In a different setup, illustrated in Fig. 2, the yeast is grown on a $0.3-2 \mathrm{~mm}$ agarose film suspended on a Millipore TCTP14250 membrane (with $10 \mu \mathrm{m}$ pores) over a continuously replenished and stirred YPD solution. At the membrane, an equilibrium between waste products (metabolites) and nutrients in the reservoir and in the agarose film is established. As seen from Figs. 1(b) and $1(\mathrm{c})$, where the front is shown at $1 \mathrm{~h}$ intervals, this setup allows for the continued growth of the front, and offers a tool to study dynamic instabilities in growing colonies.

Metabolites formed by the yeast during the degradation of nutrients may contain inhibitory elements. This is in accordance with our experimental observations: when there is no reservoir, the characteristic time for a metabolite to disappear is long and a high concentration of metabolites builds up. This was the situation shown in Fig. 1(a) where the growth of the front slowed down significantly. When growing in the replenishing setup with a thin agarose film, the metabolites diffuse through the membrane and disappear on a time scale comparable to the yeast growth rate. This is the case in Fig. 1(b), where the colony continues to grow, forming a characteristic pattern with deep grooves around local accumulations of metabolites. When growing on a thin agarose film, as shown in Fig. 1(c), there are no deep grooves, but the front evolves through a tip splitting dynamics [21].

In a standard setup, as in Fig. 1(a), only the beginning of the tip splitting dynamics is seen. One might 
(a)
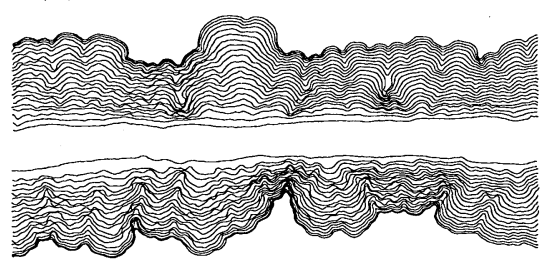

(b)
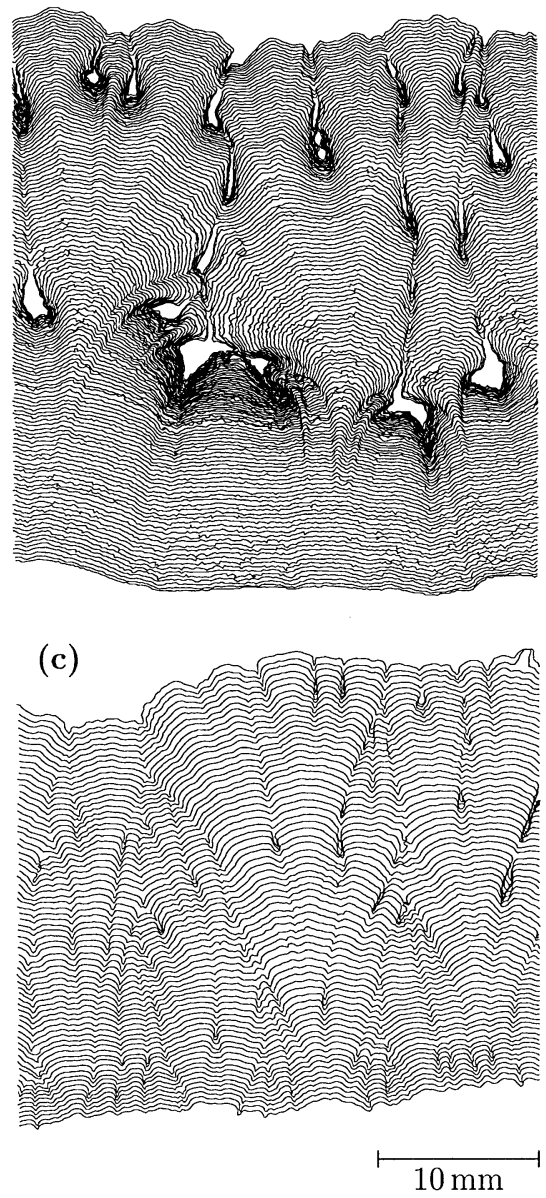

FIG. 1. Time series showing the growth of Pichia membranaefaciens at $T=30{ }^{\circ} \mathrm{C}$ and $100 \%$ relative humidity. In (a) growth is directed from center and outwards, in (b) and (c) growth is directed upwards. (a) On solid agarose: $1 / 2$ strength YPD, $3 \mathrm{~mm} 0.8 \%$ agarose film, in 5-h intervals. (b) Replenishing setup: $2.6 \mathrm{~mm}, 1 / 2$ strength YPD, $0.8 \%$ agarose film, 1/16 strength replenished YPD, in 1-h intervals. (c) Replenishing setup: $1.0 \mathrm{~mm}, 1 / 2$ strength YPD, $0.8 \%$ agarose film, 1/4 strength replenished YPD, 1-h intervals.

thus be tempted to misinterpret the coherent sectors developing along the interface as a sign of clonal variation in the yeast. However, in the replenishing setup in Figs. 1(b) and 1(c), we see that the dynamics of the morphology is indeed governed by tip splittings. Notably, the morphology does not change during the growth, thus in-
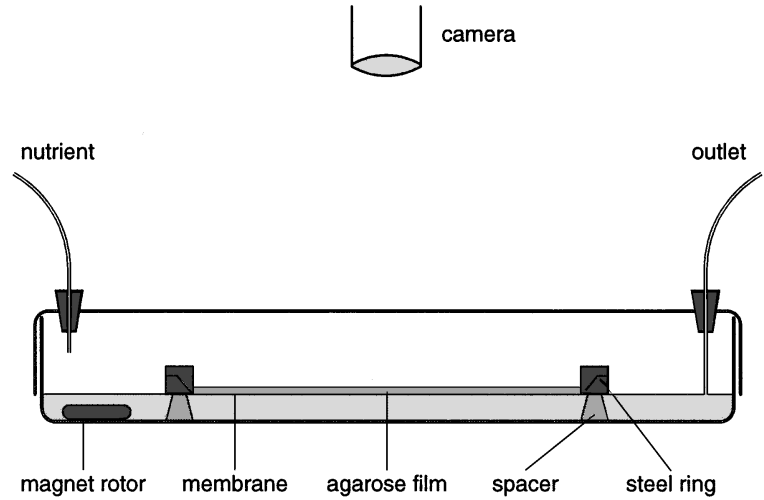

FIG. 2. Schematic view of the experimental setup with replenishing. An agarose film of thickness $0.3-2 \mathrm{~mm}$ is suspended on a membrane over a continuously replenished and stirred YPD solution. With this setup, no retardation of the growth is observed.

dicating that the structures are not caused by hereditary variation.

In order to describe the observed morphological dynamics theoretically, we build upon knowledge obtained for growth phenomena in a completely different environment. When an undercooled melt of a metal is subjected to a temperature gradient, the metal crystallizes from the cold side of the setup, i.e., directional solidification [2124]. Ahead of the crystallizing front is a field of impurities diffusing in the melt. The impurity field influences substantially the morphology of the growing front. By analogy, this is similar to what happens in the growth zone of the yeast colony where the metabolites now play the role of the impurities.

For the modeling we introduce two fields: the yeast field $w(\boldsymbol{x}, t)$ which monitors the local presence of yeast (when $w>0$ the cite $\boldsymbol{x}$ is occupied), and the metabolite field $C(\boldsymbol{x}, t)$. The two fields couple by having $w$ as a source for $C$, and $C$ suppressing the growth of $w$. The metabolite field $C$ evolves according to

$$
\frac{d C(\boldsymbol{x}, t)}{d t}=D \Delta C(\boldsymbol{x}, t)-\frac{1}{\tau} C(\boldsymbol{x}, t)+\frac{1}{h} \theta(w(\boldsymbol{x}, t)),
$$

where $D$ is a diffusion constant of the order $1 \mathrm{~mm}^{2} / \mathrm{h}$, $\tau \approx h^{2} / D$ is the characteristic time for metabolites to disappear through the agarose film, and $h$ is the thickness of the film. In the following we call $\tau$ the penetration time. The metabolite source term $\theta$ is set to unity where there is yeast present [i.e., $w(\boldsymbol{x}, t)>0$ ] and zero elsewhere.

The front of the yeast colony evolves with a normal velocity given by

$$
\boldsymbol{v}(\boldsymbol{x}, t)=\boldsymbol{n} F(C(\boldsymbol{x}, t))\langle\theta(w(\boldsymbol{x}, t))\rangle_{\ell}[1+\eta(\boldsymbol{x})],
$$

where the vector $\boldsymbol{n}$ is a unit vector along the normal of the front. $F(C)$ is a decreasing function of $C$ representing the 
suppression of the cell division from the metabolites. $\theta$ is averaged over a length scale $\ell$ related to the surface tension of the immersing liquid. $\eta$ is a Gaussian uncorrelated quenched noise with zero mean. The quenched noise represents inhomogeneities in the film. We limit our discussion to the quenched case and do not discuss a possible annealed part representing temporal fluctuations in the local population dynamics of the yeast colony.

The simulations are performed on a square lattice, simultaneously updating Eqs. (1) and (2) with time steps sufficiently small to ensure the stability of the diffusion equation. In order to implement Eq. (2), each lattice site is assigned a random number $\eta(\boldsymbol{x})$ between $-1-\Gamma$ and $-1+\Gamma$, and the yeast field $w(\boldsymbol{x}, t)$ is initially set equal to $\eta(\boldsymbol{x})$ (note that $\eta<0$ ). At each time step we consider invasion of yeast in all lattice sites which are nearest neighbors to the growing colony, meaning that the field $w$ for all border sites is incremented by an amount

$$
\frac{d w(\boldsymbol{x}, t)}{d t}=F(C) n_{n n}(\boldsymbol{x}, t)\langle\theta(w(\boldsymbol{x}, t))\rangle_{\ell} .
$$

When $w$ takes a value greater than 0 the site is invaded, and in the next time step new neighbors then start to be invaded. In Eq. (3) $n_{n n}(\boldsymbol{x})$ determines the number of nearest neighbors occupied by yeast. $\langle\theta(w(\boldsymbol{x}, t))\rangle_{\ell}$ models the surface tension at $\boldsymbol{x}$ and is set equal to the fraction of points within a certain length scale $\ell$ from $x$ which have already been fully occupied by the advancing colony. $F(C)$ is assumed to equal $\exp \left(-\sqrt{C / C_{0}}\right)$ with $C_{0}=0.25$, a form which is chosen to reproduce the observed pattern sensitivity of the agarose thickness. This functional form is an assumption; one could also use other forms like exponential, linear, or power law decay. We have used various functions in the simulations, and in all cases where the decay of $F$ versus $C$ is not too weak we observe qualitatively the same results as will be presented here.

In our phenomenological model a number of parameters enter. Some can be scaled out and others are under experimental control. In summary there are three important parameters: the coupling strength to the metabolic field, determined effectively by $F$, the penetration time $\tau$, and the strength of the noise $\Gamma$. In addition, there is the diffusion constant $D$ which is fixed by the size of the metabolite to be of order $1 \mathrm{~mm}^{2} / \mathrm{h}$ (equal that of a protein in water), and there is the surface tension scale $\ell$ which determines our lower cutoff, and is connected to the surface tension of the carrier liquid.

First let us consider two extreme cases of the model. Case one is the limit where there is no coupling to the metabolic field. Then the envelope of the yeast colony will grow as a random deposition model, exhibiting a rough surface on scales larger than $\ell$ with statistics as the Eden growth model in statistical physics [25]. In case two, we consider the situation where there is no noise $\Gamma=0$. In that case structures can only develop from initial inhomogeneities. For monotonically decreasing $F$, the front is destabilized according to Eqs. (1) and (2) because parts of the front which are left behind experience a stronger metabolic field and accordingly slow down. In Fig. 3(a) we see an example of the front evolution without noise in the unstable regime of the model.

To model the experimental findings shown in Figs. 1(b) and $1(\mathrm{c})$, we investigate the model with diffusion constant $D=1$, and the length over which the front is averaged $\ell=2$ (in units of lattice spacing). We vary $h$ and set $\tau=$ $h^{2} / D$ and use $h=8$ in Figs. 3(a) and 3(b), and $h=3$ in 3(c). In Figs. 3(a) and 3(b) we simulate 6000 time steps; in 3(c) we only simulate 2000 time steps. In Fig. 3(a) the noise parameter is set to $\Gamma=0$ and the simulation is initialized with a front modulated by a long wavelength perturbation. One then observes a tip splitting dynamics with deep grooves and overhangs. However, in order to reproduce the experimentally observed irregularities we introduce noise in Figs. 3(b) and 3(c) by setting $\Gamma=$ 0.5. In Fig. 3(b), where $h=8$, a morphology with large grooves which develop as the front circumvents local accumulations of metabolites is seen. These grooves are due to the fact that the metabolite field locally hinders the growth for a while. As time progresses, however, the colony grows around the place where the growth slowed down and the colony may then recombine again atop of the place with high $C$ region. There is qualitative agreement with the experiment shown in Fig. 1(b).

In the final case, seen in Fig. 3(c) where $h=3$, to be compared with Fig. 1(c), a compact morphology is observed. The thinner agarose layer means that the metabolites more easily disappear through the membrane, thus leading to a weaker instability without deep grooves and overhangs. However, the instability is still present and manifests itself in terms of a continued tip splitting dynamics where individual valley exhibits long temporal memory. The pattern is similar to the one obtained by a Kuramoto-Sivashinsky equation in $1+1$ dimension $[26,27]$, a connection that may be formalized by a linear stability analysis of our model.

In conclusion, we have presented experimental and theoretical studies of the growth morphologies of colonies of the yeast Pichia membranaefaciens. As a function of a control parameter, which in the experimental case is the thickness of the agarose plate, very different morphologies are observed. These range from a "cellular" front with penetrating instabilities for a large agarose plate thickness, over a morphology with deep grooves, to a compact colony which exhibits tip splitting instabilities but no overhangs or grooves. As a model we have introduced a front dynamics governed by a coupling to an inhibitory diffusive field, and have observed qualitative agreement. We believe that the model could be applied at other parameter ranges than utilized here, and that the model can be used to extrapolate and analyze morphologies at similar conditions for other types of growing colonies. 


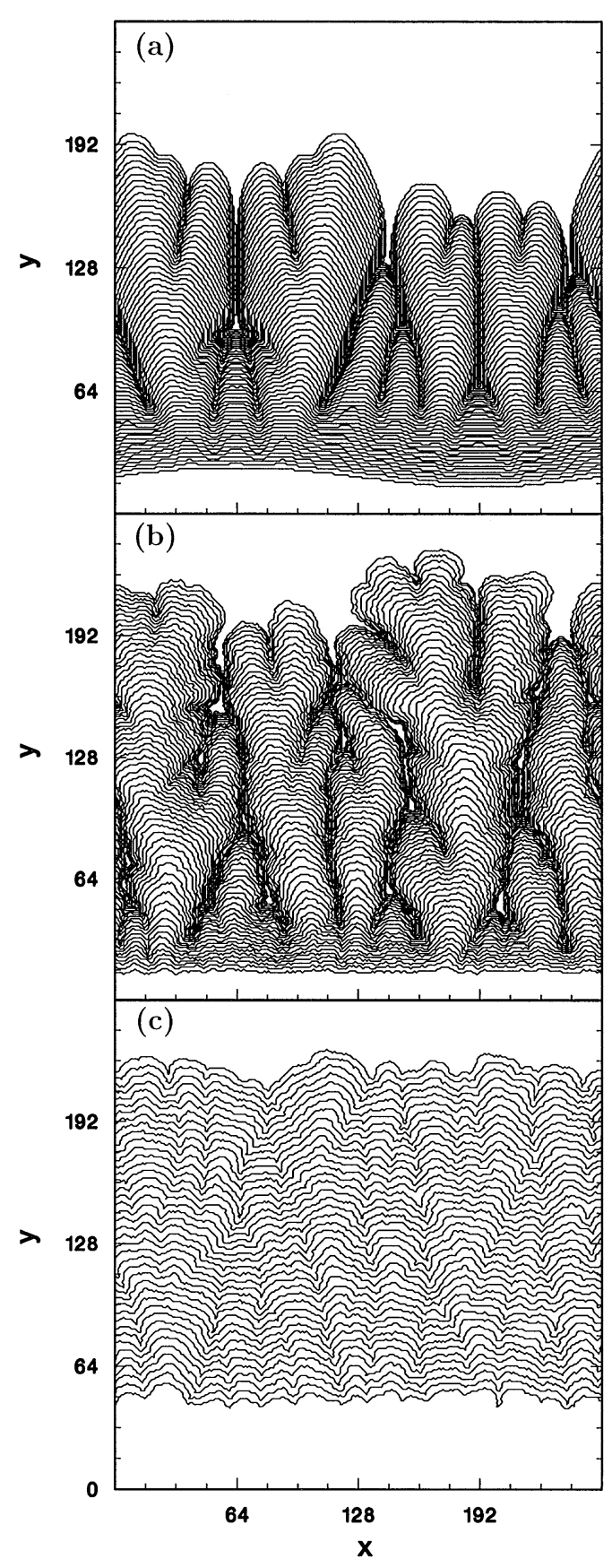

FIG. 3. Time series of the fronts obtained from simulations of the model defined by Eqs. (1) and (2). The following parameters are used: $D=1, C_{0}=0.25$, and $\ell=2$. Further in (a) thickness $h=8$ and noise level $\Gamma=0$; (b) $h=8, \Gamma=$ 0.5 ; (c) $h=3, \Gamma=0.5$. In cases (a) and (b) the simulation time is 6000 time steps and 2000 time steps in (c). Initial conditions: In (a), $w>0$ below a sine wave of amplitude 5 and wavelength 256 . In (b) and (c), $w>0$ below a horizontal line.

We gratefully acknowledge the advice from F. Andreasen, T. Bohr, K. B. Pedersen, V. Putkaradze, and Y.C.
Zhang during the project. We would like to thank especially $\mathrm{T}$. Bohr for providing financial support from the Hallas Møller foundation.

*Electronic address: mhjensen@nbi.dk

[1] D'Arcy Thompson, On Growth and Form (Cambridge University Press, Cambridge, 1942).

[2] A. M. Turing, Philos. Trans. R. Soc. London B 237, 37 (1952).

[3] K. J. Lee, E. C. Cox, and R. E. Goldstein, Phys. Rev. Lett. 76, 1174 (1996).

[4] R. E. Goldstein, Phys. Rev. Lett. 77, 775 (1996).

[5] J. O. Kessler, Commun. Theor. Biol. 212, 85 (1989).

[6] N. A. Hill, T. J. Pedley, and J. O. Kessler, J. Fluid Mech. 208, 509 (1989).

[7] J. A. Shapiro and D. Trubatch, Physica (Amsterdam) 49D, 214 (1991).

[8] J. A. Shapiro, BioEssays 17, 597 (1995).

[9] J. Wakita, K. Komatsu, A. Nakahara, T. T. Matsuyama, and M. Matsushita, J. Phys. Soc. Jpn. 63, 1205 (1994).

[10] Y. Shimada, A. A. Nakahara, M. Matsushita, and T. Matsuyama, J. Phys. Soc. Jpn. 64, 1896 (1995).

[11] E. Ben-Jacob, O. Schochet, A. Tenenbaum, I. Cohen, A. Cziròk, and T. Vicsek, Nature (London) 368, 46 (1994).

[12] E. Ben-Jacob, A. Tenenbaum, O. Shochet, and O. Avidan, Physica (Amsterdam) 202A, 1 (1994).

[13] J. W. Crawford and I. M. Young, Geoderma 1-4, 157 (1993).

[14] D. B. Patankar and T. Oolman, Biotechnol. Bioeng. 42, 571 (1993).

[15] J. A. Soddell and R. J. Seviour, Binary 6, 21 (1994).

[16] S. Matsuura and S. Miyazima, Physica (Amsterdam) 30A, 191 (1992).

[17] S. Matsuura and S. Miyazima, Fractals 1, 11 (1993).

[18] M. J. Carlile and S.C. Watkinson, The Fungi (Academic Press, London, 1994).

[19] Pichia membranaefaciens E. C. Hansen was determined by Dr. P. Hoffman, Deutsche Sammlung von Mikroorganismen und Zellkulturen, 1995.

[20] A. P. J. Trinci, J. Gen. Microbiol. 67, 325 (1971).

[21] J. S. Langer, Rev. Mod. Phys. 52, 1 (1980).

[22] M. Kertzberg, Phys. Rev. B 27, 3909 (1983).

[23] J. Bechhoefer and A. Libchaber, Phys. Rev. B 35, 1393 (1987).

[24] S. Sarkar and M. H. Jensen, Phys. Rev. A 35, 1877 (1987).

[25] T. Halpin-Healy and Y.-C. Zhang, Phys. Rep. 254, 215 (1995).

[26] Y. Kuramoto, Chemical Oscillations, Waves and Turbulence (Springer, Berlin, 1984); Y. Kuramoto, Suppl. Theor. Phys. 64, 346 (1978); G. I. Sivashinsky, Acta Astron. 4, 1177 (1977).

[27] K. Sneppen, J. Krug, M. H. Jensen, C. Jayaprakash, and T. Bohr, Phys. Rev. A 46, R7351 (1992). 